\title{
Primer Registro de la Avoceta Americana (Recurvirostra americana) para el Sur de Honduras
}

\author{
Mario Espinal $^{1}$ y José Manuel Mora ${ }^{2}$
}

Resumen. La avoceta americana (Recurvirostra americana) se distribuye desde la parte central-sur de Canadá hasta el centro y otros sectores de los Estados Unidos de América, México y Centroamérica. En Honduras, la avoceta es un raro visitante de invierno con registros muy escasos en la costa norte del país. El 28 de octubre de 2009, observamos y fotografiamos dos individuos de esta especie alimentándose en las orillas de la laguna de invierno Guameru, Choluteca $\left(13^{\circ} 06^{\prime} 25.82^{\prime \prime} \mathrm{N}\right.$ y $87^{\circ}$ 09'30.87' O). Ambas aves tenían un plumaje no reproductivo con la cabeza y el cuerpo blanco, y las alas negras. Este ese el primer registro documentado de Recurvirostra americana para el departamento de Choluteca y todo el Sur de Honduras. Esta observación resalta la necesidad de realizar inventarios de la biodiversidad en la región costera del Golfo de Fonseca y otros sitios sensibles de Honduras.

Palabras clave: Ampliación ámbito de distribución, especie de consideración especial, SIEPAC, visitante de invierno.

First Record of the American Avocet (Recurvirostra americana) in Southern Honduras

\begin{abstract}
The American Avocet (Recurvirostra americana) is found from central-southern Canada to central and other parts of the United States of America, Mexico and Central America. In Honduras the American Avocet is a rare winter visitor with very few records on the northern coast of the country. On October 28, 2009 we observed and photographed two individuals of this species feeding on the Guameru winter lagoon, Choluteca (13 ${ }^{\circ} 0625.82$ " N and $87^{\circ} 0930.87$ " W). Both birds had non-breeding plumage with a white head and body and black wings. This is the first documented record for the American Avocet for Choluteca department and throughout all southern Honduras. This observation highlights the need for inventories of biodiversity in the coastal region of the Gulf of Fonseca and other Honduras sensitive sites.
\end{abstract}

Key words: distribution range expansion, SIEPAC, special consideration species, winter visitor.

\section{Introducción}

Las aves de la familia Recurvirostridae son de tamaño mediano $(30-50 \mathrm{~cm})$, esbeltas, con predominio del color negro y blanco en su plumaje, con patas y picos largos. Las especies del género Recurvirostra tienen el pico recurvado hacia arriba. La familia tiene siete especies en el mundo (Canevari et al. 2001), de las cuales se han registrado a Himantopus mexicanus y a Recurvirostra americana en Honduras (AOU 2010).

La avoceta americana se distribuye desde la parte central-sur de Canadá al centro de Estados Unidos (donde se reproduce) hasta otros sectores de los Estados Unidos y México (donde migra) (Brinkley 2008). Asimismo, se ha reportado en Guatemala (Eisermann 2006), Honduras (Thorn et al. 2006), El
Salvador (Herrera et al. 2006), Nicaragua (ZolotoffPallais et al. 2009), Costa Rica (Alvarado 2006) y Panamá (Angehr 2005).

En Honduras, la avoceta americana es un raro visitante de invierno (Monroe 1968). Se ha reportado de manera esporádica en la costa norte del país, donde se han encontrado pocos individuos (3-8 individuos). Debido a ello, en Honduras se ha clasificado como una especie de "Consideración Especial", según la clasificación oficial del país (Thorn et al. 2006). Ésta categoría se refiere a que la especie no está en peligro en Honduras, pero es escasa y hay que ponerle atención. La observación más reciente de la especie fue el 23 de junio de 2012 en Cabo Camarón, departamento de Gracias a Dios por D. Germer y E. López, también en el norte del país (ebird.org. 2013).

\footnotetext{
${ }^{1}$ Investigador Asociado, Centro Zamorano de Biodiversidad, Escuela Agrícola Panamericana, Honduras. Correo electrónico mknorops@gmail.com

2 Instituto Internacional en Conservación y Manejo de Vida Silvestre (ICOMVIS), Universidad Nacional (UNA), Heredia, Costa Rica. Correo electrónico josemora07@gmail.com
}

DOI: $10.5377 /$ ceiba.v52i2.1719 


\section{Descripción y Discusión}

El 28 de octubre de 2009, en el área de trabajo para la construcción de la línea de transmisión eléctrica del proyecto Sistema de Interconexión Eléctrica para los Países de América Central (SIEPAC), se observaron $y$ fotografiaron dos individuos de Recurvirostra americana. Estas aves tenían un plumaje característico de los individuos que no están en reproducción, tenían la cabeza y el cuerpo de color blanco, y las alas de color negro (Figura 1). Ambos individuos formaban parte de un grupo mixto de aves playeras que estaban alimentándose en el borde de la laguna de invierno Guameru, en la carretera que conduce hacia la camaronera Granjas Marinas San Bernardo en el departamento de Choluteca (13 ${ }^{\circ} 06^{\prime} 25.82^{\prime \prime} \mathrm{N}$ y $87^{\circ} 09^{\prime} 30.87^{\prime \prime}$ O) en el Sur de Honduras. Las dos avocetas fueron observadas en la mañana junto a algunos individuos de la garza tricolor (Egretta tricolor), tringa patas amarillas (Tringa melanoleuca) y varios soldados (Himantopus mexicanus) (Figura 2). Esta observación es el primer registro documentado de Recurvirostra americana para el departamento de Choluteca y todo el Sur de Honduras.

Jenner et al. (2007) proporcionaron una lista de las especies de aves de posible existencia en la región costera del pacífico del Sur de Honduras. Aunque la lista incluyó a la avoceta americana, esta especie no ha sido observada con anterioridad a este reporte en ninguna localidad del Sur de Honduras. Este reporte es el primer registro documentado de la avoceta americana para el departamento de Choluteca y todo el Sur de Honduras y es a la vez apenas el tercero para el país. No obstante, hay siete observaciones recientes en eBird, también del Sur de Honduras, pero posteriores a su primer avistamiento aquí reportado.

La presencia de esta especie enfatiza la necesidad de inventariar la biodiversidad en la región Costera del Golfo de Fonseca. A la vez, sería recomendable observar y, de ser posible, monitorear para contar con más información sobre la avoceta americana, de su estado poblacional y de su distribución en Honduras.

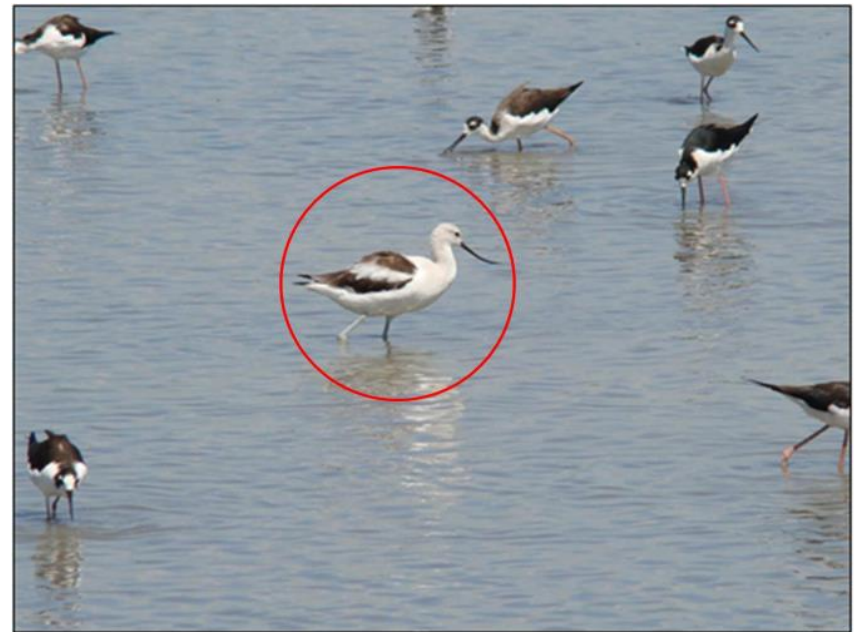

Figura 1. Individuo de Recurvirostra americana (en el círculo), acompañado de un grupo de individuos de Himantopus mexicanus. Laguna de invierno Guameru, Choluteca, Honduras. Fotografía de Mario Espinal tomada, el 28 de octubre de 2009.

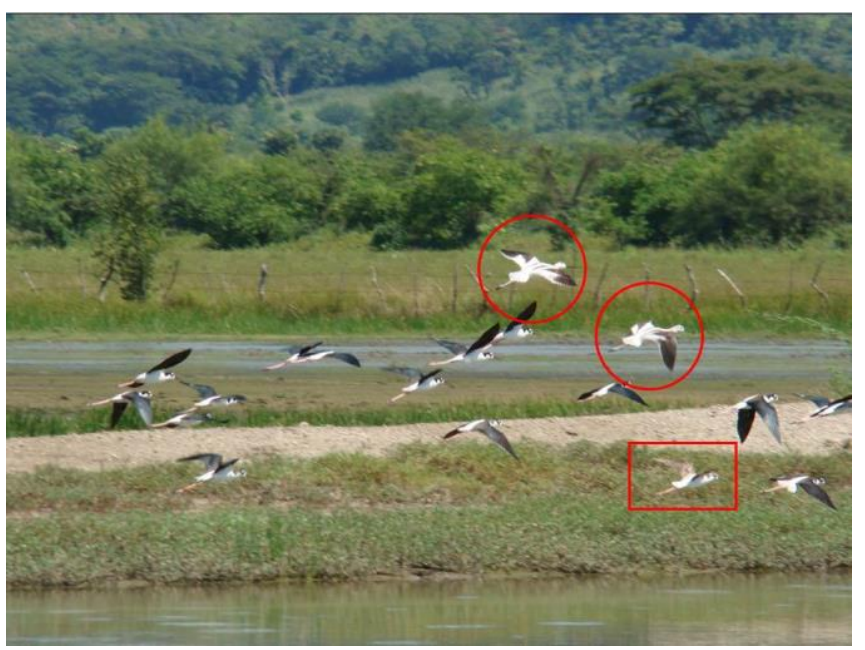

Figura 2. Dos individuos de Recurvirostra americana (círculos) en vuelo, acompañados por un grupo de Himantopus mexicanus y un individuo de Tringa melanoleuca (rectángulo). Laguna de invierno Guameru, Choluteca, Honduras. Fotografía de Mario Espinal, tomada el 28 de octubre de 2013. 


\section{Agradecimientos}

Mario Espinal agradece a Eléctricas de Medellín S.A. (EDEMSA) por la oportunidad de trabajar en el Proyecto SIEPAC-Honduras. Ambos autores agradecen a Daniel Germer de la Asociación Hondureña de Ornitología (ASHO), por sus sugerencias para la preparación del manuscrito.

\section{Literatura Citada}

Alvarado, G. 2006. Reporte Final Aves Acuáticas en Costa Rica, Departamento de Historia Natural. Museo Nacional de Costa Rica. 77 p.

American Ornithologists' Union (AOU). 2010. Séptima Edición, (Incluyendo Suplemento 51a) Nomenclatura para Honduras. $26 \mathrm{p}$.

Angehr, G. 2005. Final Report Waterbirds in Panamá, Panamá Audubon Society- Waterbirds Conservation for the Americas. $146 \mathrm{p}$.

Brinkley, E.S. 2008. Field Guide to Birds of North América, National Wildlife Federation, Sterling/New York/London. 527 p.

Canevari, P., G. Castro, M. Sallaberry y L. Naranjo. 2001. Guía de los Chorlos y Playeros de la Región Neotropical. American Bird Conservancy, WWW-US, Humedales para las Américas y Manoment Conservation Science, Asociación Calidris. Santiago de Cali, Colombia. 141 p.
eBird. 2013. An online database of bird distribution and abundance [web application]. eBird, Cornell Lab of Ornithology, Ithaca, New York. Available: http://www.ebird.org. (Accedido el 20 de septiembre de 2013).

Eisermann, K. 2006. Part of an Evaluation of Waterbird Populations and their Conservation in Guatemala, Sociedad Guatemalteca de Ornitología, Waterbird Conservation for the Americas. $74 \mathrm{p}$.

Herrera, N., O. Komar y R. Ibarra Portillo. 2006. Reporte Final Aves Acuáticas en El Salvador, SalvaNATURAWaterbirds Conservation for the Americas. $57 \mathrm{p}$.

Jenner, T., O. Komar y A. Narish. 2007. Noteworthy bird records from the Gulf of Fonseca, Honduras. Cotinga 28:13-20.

Monroe, B.L. Jr. 1968. A distributional survey of the birds of Honduras. Ornithological Monographs 7: 1-458.

Thorn, S., D. Medina y D. Shoch. 2006. Reporte Final Aves Acuáticas en Honduras. Waterbird Conservation for the Americas. $36 \mathrm{p}$.

Zolotoff-Pallais, J.M., M. Lezama y W. Arendt. 2009. Estado de Conservación de Aves Acuáticas y sus Hábitats en Nicaragua, USAID, Fundación COCIBOLCA, US Forest Service. 92 p.

Recibido para publicación el 18 de noviembre de 2013. Aceptado para publicación el 18 de abril de 2014. 